\title{
A simple model for rapid gypsum determination in arid soils
}

\author{
El-Sayed E. Omran ${ }^{1}$
}

Received: 19 August 2016/ Accepted: 18 October 2016/Published online: 27 October 2016

(C) Springer International Publishing Switzerland 2016

\begin{abstract}
The development of a reliable, fast and inexpensive method to determine the gypsum content in soils to understand their behavior is crucial. However, current methods of determination are cumbersome, lengthy and imprecise. This study was conducted to develop a rapid and simple method to determine soil gypsum content. A gypsum content of 120 soil samples was determined by three methods; (1) standard silica-gel; (2) acetone; and (3) the proposed method (OMRAN GypSim). These methods were further tested for the recovery of known amounts of pure gypsum (over the range of 0-100\%). The proposed method estimates the gypsum content of water loss in the soil sample by heating at $70{ }^{\circ} \mathrm{C}$ and $135-150{ }^{\circ} \mathrm{C}$. Sandy and clay minerals mixed with gypsum reagent powder (over the range of $0-100 \%$ gypsum) were used as a reference. Gypsum $\left(\mathrm{CaSO}_{4} \cdot 2 \mathrm{H}_{2} \mathrm{O}\right)$, after heating at $150{ }^{\circ} \mathrm{C}$, dehydrates, losing the two water molecules and becoming mostly anhydrite $\left(\mathrm{CaSO}_{4}\right)$. The crystal water of gypsum is not lost when the gypsum is dried at $70{ }^{\circ} \mathrm{C}$ for $45 \mathrm{~min}$., whereas it is lost when the gypsum is heated at $150{ }^{\circ} \mathrm{C}$ for $15 \mathrm{~min}$. The reproducibility of the proposed method is worth highest (average 0.10) followed by silica-gel (average 0.35) and acetone (average 0.47). This simple, yet effective methods determined gypsum content to within $1 \%$, whereas other methods exceeded gypsum content by as much as $10 \%$. An easy-to-use graphical user interface has been designed for the model running in a user-friendly way.
\end{abstract}

El-Sayed E. Omran

ee.omran@gmail.com

1 Soil and Water Department, Faculty of Agriculture, Suez Canal University, Ismailia 41522, Egypt
Keywords Gypsum - Silica-gel · Crystal water · Hygroscopic water · Prediction, Modeling

\section{Introduction}

Gypsum is widespread in both arid and semi-arid regions. Gypsum $\left(\mathrm{CaSO}_{4} \cdot 2 \mathrm{H}_{2} \mathrm{O}\right)$ is found over a wide range of temperatures, but most gypsic soils occur in xeric, ustic, and aridic soil moisture regimes (FAO 1990). It is the most common sulfate mineral in soils. Gypsum has several undesirable effects due to its solubility. A concentration of $>10 \%$ gypsum significantly interferes with soil characteristics such as structure, consistency, and water-holding capacity, while soils with more than $25 \%$ gypsum do not provide a good medium for plant growth. As a calciumbased mineral, gypsum inhibits the uptake of zinc, magnesium, iron, copper, and phosphorus in plants, resulting in nutrient deficiencies. The cation-exchange capacity is reduced, and thus the level of fertility is lowered. Reprecipitation of gypsum can result in root and water restrictions in the soil and can cause increased salinity above a gypsum pan. The somewhat high solubility of gypsum increases the osmotic potential of the material. As a result, the availability of water to plants can be decreased. Gypsum has also a considerable influence on soil properties (Visconti et al. 2010). When it is the main component of the soil, it controls its behavior. Some gypsiferous soils have been characterized by utility limitations such as the sinkholes formation (Omran 2012; Reda 1995). High water table can dissolve gypsum in the soil. Irrigation in areas with gypsum also can result in dissolution. The material goes into solution or easily erodes and forms solution cavities, pipes, and gullies. Gypsum can go into solution and then reprecipitate. Soil gypsum occurs in various forms 
in the field, according to the mode of formation (Reda 1995): small and large crystals, veins, soft powder or spots, patches, segregations or thread-like, and gypsum layer. An accurate measurement of gypsum in soil is very important because it affects many soil properties, which in turn influence management, classification, and suitability of the soils. Therefore, it is necessary to quantify accurately the amounts of gypsum in soils.

A review of the literature reveals that the previous theoretical or experimental work, which has been undertaken to determine the gypsum content in soils is rare. In terms of theoretical modelling, current methods of gypsum determination in soils, which are cumbersome and imprecise (Lebron et al. 2009), could be grouped into; wet chemical; X-ray diffraction and loss of crystal water (Farag 1999). Wet chemical methods are laborious and subject to errors due to cation exchange and occlusion of dissolved salts in precipitation (Skarie et al. 1987; Artieda et al. 2006). Also, wet methods are unsuitable for soils rich in gypsum or containing other sulfates (Herrero et al. 2016). Mahabadi and Givi (2008) evaluate different methods of gypsum determination, which the method of sodium carbonate is the most accurate one. Acetone, calcium magnesium difference, and diluted extracted sulfate methods are not sufficiently accurate. The acetone method could be considered the most reliable but very lengthy (Elprince and Turjoman 1983). Sulfate determinations by titrimetric, photometric, and luminescence methods requiring difficultto-access and expensive reagents for their use are extremely complex, labor-intensive, and lengthy. An X-ray diffraction technique for gypsum determination (Weindorf et al. 2014) has problems of preferred orientation of gypsum and the replicates needed for counting (Khan and Webster 1968).

Methods based on the loss of crystal water of gypsum, do not rely on $\mathrm{SO}_{4}$ determinations and thus estimate the gypsum content without interference by other sulfate minerals contained in the soil sample. The thermogravimetric methods of gypsum rely on the measure of the crystal water content of gypsum. Typically, the value $20.91 \%(\mathrm{w} / \mathrm{w})$ is used; however, because not all the water is recovered at the heating temperatures used in the laboratories. The concept of a recovery $(19.42 \%)$ factor (Burns et al. 2002) is useful when describing the analytical methods based on the loss of crystal water. (Nelson et al. 1978) realized the limitations of the method in low gypsum content soils, which minimizes the usefulness of the measure for taxonomic purposes, considering that the threshold for the gypsic horizon stated by Soil Survey Staff (2010) is 5\% gypsum content. Elprince and Turjoman (1983) realized that the silica-gel method seems simple but slow in the proceeding. When the sample is heated to $105{ }^{\circ} \mathrm{C}$, part of the crystal water of gypsum $\left(\mathrm{CaSO}_{4} \cdot 2 \mathrm{H}_{2} \mathrm{O}\right)$ is lost, and bassanite $\left(\mathrm{CaSO}_{4} \cdot 0.5 \mathrm{H}_{2} \mathrm{O}\right)$ is produced. With increasing temperatures, all gypsum is transformed into anhydrite $\left(\mathrm{CaSO}_{4}\right)$.

All these methods have their limitations and advantages, but most are associated with large errors (Porta 1998). AlAwajy et al. (1994) criticized all these methods and recommended none of them to be the best. So, traditional gypsum determination methods, which are lengthy, laborintensive, and insufficiently accurate appear to be increasingly irrelevant to many users and does not have a market for land managers and policy makers (Omran 2008). A reliable and environmentally friendly method is needed to rapidly detect and analyze soil gypsum. A growing demand for the development of more timely and cost-effective methods for quantifying gypsum in soil with reliable precision, lead us to a research question: Can we develop a more accelerated, simple, and accurate measure replace the out-of-date techniques for soil gypsum determination and suitable for soils with gypsum content as low as $5 \%$ ?

\section{Thermal changes of gypsum}

Calcium sulfate occurs in three different hydrates: dihydrate (DH: $\left.\mathrm{CaSO}_{4} \cdot 2 \mathrm{H}_{2} \mathrm{O}\right)$, hemihydrate $\left(\mathrm{HH}: \mathrm{CaSO}_{4-}\right.$ $\left.0.5 \mathrm{H}_{2} \mathrm{O}\right)$, and anhydrite $\left(\mathrm{AH}: \mathrm{CaSO}_{4}\right)$. The thermal increases the gypsum transformation into bassanite $\left(\mathrm{CaSO}_{4} \cdot 0.5 \mathrm{H}_{2} \mathrm{O}\right)$. When soil with $50 \%$ gypsum in its fine earth fraction was heated at $205^{\circ} \mathrm{C}$, it is partly changed into bassanite. Bassanite could not be found in the samples heated at $105{ }^{\circ} \mathrm{C}$, but it was in those heated at $205^{\circ} \mathrm{C}$ (León et al. 2014). When the gypsum is heated up to $105{ }^{\circ} \mathrm{C}$, only a small fraction (13-19\%) of gypsum is removed. Subsequent partial rehydration in the laboratory results in the formation of bassanite at relative moisture below saturation (Lebron et al. 2009; León et al. 2014). At $105{ }^{\circ} \mathrm{C}$, the gypsum crystal loses $13-19 \%$ of its mass (Artieda et al. 2006), corresponding to the two water molecules. Lebron et al. (2009) concluded that the temperature at which total water disappears is around $163{ }^{\circ} \mathrm{C}$, since fast heating does not allow enough time for the water to diffuse through the crystal. Therefore, in the mineralogical analysis bassanite did not appear until temperatures achieved $205^{\circ} \mathrm{C}$ (León et al. 2014). The transition temperature for the change from gypsum to anhydrite when in contact with water is provisionally placed at $38{ }^{\circ} \mathrm{C}$.; for the change from gypsum to hemihydrate, when in contact with water, is most definitely placed at $98{ }^{\circ} \mathrm{C}$. Anhydrite has been obtained experimentally from gypsum in contact with water at $100{ }^{\circ} \mathrm{C}$. It is confirmed that the only two solids existing in the system between 0 and $200{ }^{\circ} \mathrm{C}$ are gypsum and anhydrite. 


\section{Materials and methods}

\section{Soil samples and experimental materials}

One-hundred and twenty (120) soil samples, which have been collected from the most common great groups at various sites in Egypt (Fig. 1) were air-dried and passed through a $2 \mathrm{~mm}$ sieve. Reconstituted gypsum soil samples were made from non-gypsiferous sandy and clay soils and also naturally pure gypsum (From El-Ballah, Ismailia, Egypt) and calcium sulfate laboratory reagent (CAS 7778-18-9). Native and added gypsum in the samples were determined by two methods acetone, and silica-gel and compared with the proposed method.
1. Acetone precipitation method. This method is a wet chemical method. It includes extraction of soil sample with an amount of water sufficient to dissolve all the gypsum present and then precipitated with acetone. The precipitate is completely redissolved in distilled water and gypsum is obtained by measuring the electrical conductivity of the solution (US Salinity Laboratory Staff 1954).

2. Silica-gel method. $8.0 \mathrm{~g}$ from each soil sample were placed in an aluminum dish and dried over silica-gel in a desiccator for $48 \mathrm{~h}$ and then heated at $105^{\circ} \mathrm{C}$ for $24 \mathrm{~h}$. The differences between the silica-gel dried and oven dried sample weights gave reasonable way to determine gypsum content in the soil. Crystal water of gypsum and gypsum content of soil samples was
Fig. 1120 soil samples collected from various locations in Egypt

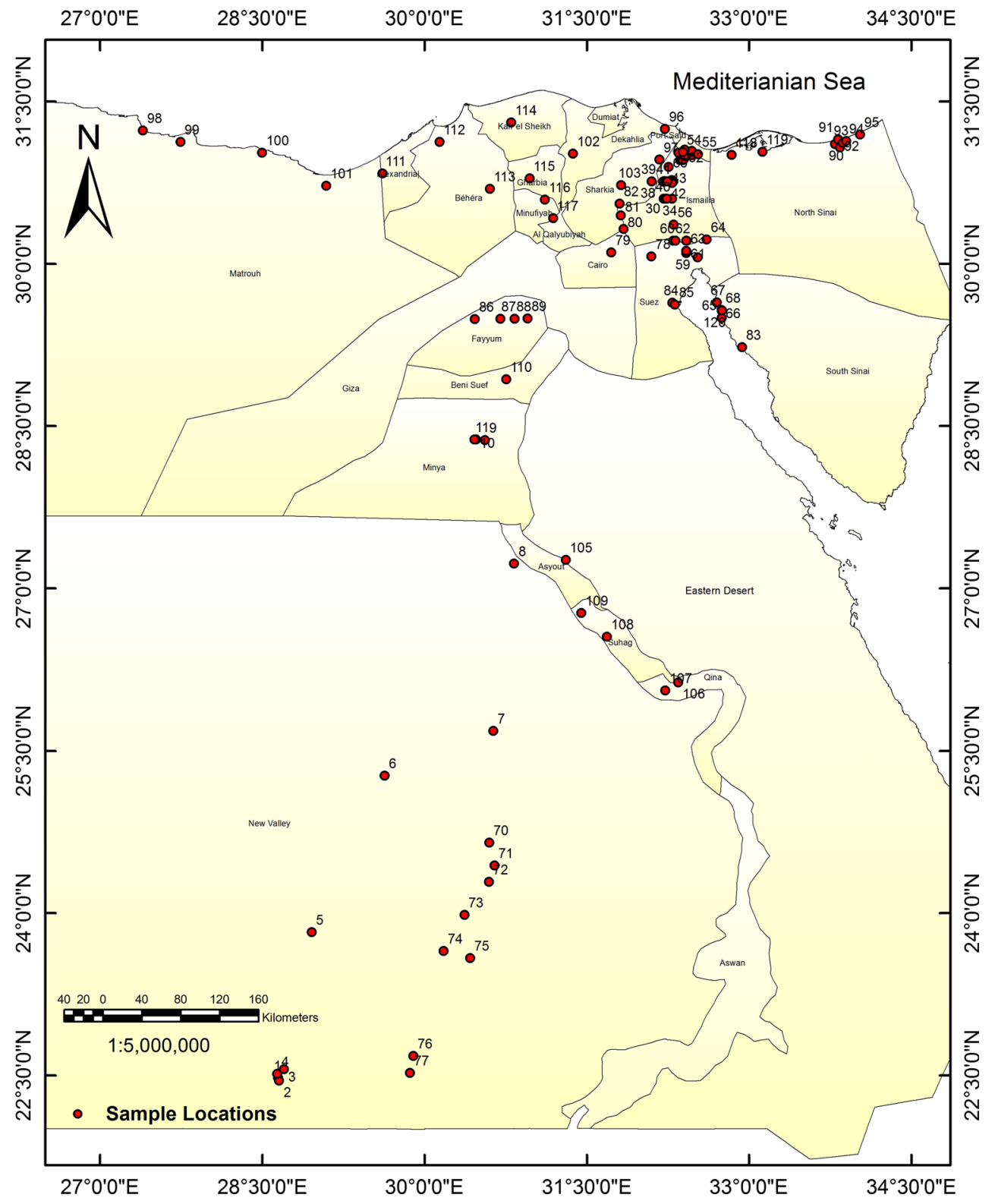


calculated according to the equation of Nelson et al. (1978).

3. The proposed accelerated method. In this method, each soil samples was placed in a Pyrex dish and dried over $70{ }^{\circ} \mathrm{C}$ in an oven for $45 \mathrm{~min}$. and then heated at $150{ }^{\circ} \mathrm{C}$ for $15 \mathrm{~min}$. The differences between the two weights gave a practical way to gypsum determination in soils.

\section{Rapid and low-cost gypsum determination method}

Low-cost gypsum determination using gypsum simulation (OMRAN GypSim) model was developed and implemented. In this method, the loss in water after heating at $70{ }^{\circ} \mathrm{C}$ for $45 \mathrm{~min}$. and after heating using different temperature degrees (over the range of $60-150{ }^{\circ} \mathrm{C}$ ) were compared. The results indicate that heating at temperature $70{ }^{\circ} \mathrm{C}$ for $45 \mathrm{~min}$. equal to silica-gel for $48 \mathrm{~h}$ and heating at temperature $105{ }^{\circ} \mathrm{C}$ for $24 \mathrm{~h}$ equal to heating at temperature $135-150{ }^{\circ} \mathrm{C}$ for $15 \mathrm{~min}$. So, for a rapid and simple method, heating at temperature 70 and $150{ }^{\circ} \mathrm{C}$ is simple, more rapid, and accurate.

The procedure for the proposed method to estimate soil gypsum as follows:

1- 10-20 g of 2-mm air-dried soil was transferred to a Pyrex dish and weighed to the nearest $0.001 \mathrm{~g}$. The dish was placed in an oven at $70{ }^{\circ} \mathrm{C}$ for $45 \mathrm{~min}$.

2- Then the dish containing the sample was placed in an oven at $150{ }^{\circ} \mathrm{C}$ for $15 \mathrm{~min}$ (the time depends on the oven volume and the number of samples put in the oven, the oven must have ventilation).

3- After the removal from the oven and before weighing, the sample was cooled completely in a desiccator. The gypsum percentage in the sample was calculated by the following equation:

$$
\begin{aligned}
\operatorname{Gypsum} \%= & \left(\mathrm{W}_{70}-\mathrm{W}_{150} / \mathrm{W}_{70}-\mathrm{W}_{\mathrm{d}}\right) \\
& * 100(100 / 19.66) \\
= & \left(\mathrm{W}_{70}-\mathrm{W}_{150} / \mathrm{W}_{70}-\mathrm{W}_{\mathrm{d}}\right) * 508.65 .
\end{aligned}
$$

where $\mathrm{W}_{70}=$ weight of the sample dried at $70{ }^{\circ} \mathrm{C}$ plus Pyrex dish, $\mathrm{W}_{150}=$ weight of the sample dried at $150{ }^{\circ} \mathrm{C}$ plus Pyrex dish, $\mathrm{W}_{\mathrm{d}}=$ weight of the Pyrex dish, and 19.66 is the recovery factor of gypsum between 70 and $150{ }^{\circ} \mathrm{C}$.

Gypsum converts slowly to hemihydrate in air at about $70{ }^{\circ} \mathrm{C}$, and more rapidly above $90{ }^{\circ} \mathrm{C}$ and at higher temperature produces anhydrite (Reda 1995). The decomposition calcium sulfate reagent dehydrate varies from 87 to $133{ }^{\circ} \mathrm{C}$ in comparison with $100{ }^{\circ} \mathrm{C}$ for the decomposition of water and produce soluble anhydrite, rather than the insoluble anhydrite, or hemihydrate (Elprince et al. 1982). By maintaining the samples at $70{ }^{\circ} \mathrm{C}$ for $45 \mathrm{~min}$., and later at $135-150{ }^{\circ} \mathrm{C}$ for $15 \mathrm{~min}$., we derived an estimate of the percentage of crystal water weight loss for this temperature interval, referred to as the weight of the dry sample at $70{ }^{\circ} \mathrm{C}$. The recovery percentage for this temperature interval was 19.66 .

\section{Checking the fit of the proposed OMRAN GypSim method}

A formal accuracy assessment involves field verification of the proposed model was performed following the procedures of Congalton and Green (1999). From a statistical perspective, the number of samples to be validated must be adequate for measuring the variability associated with the variable tested. Several approaches were proposed for testing how well the proposed model fits the data. Most approaches rely on the idea of comparing an observed number of individuals with the number expected if the fitted model were valid. These observed and expected numbers are combined to form a Chi square statistic called the goodness of fit. The model was utilized to predict gypsum values based on soil properties. Then, regressions were developed between predicted values and actual gypsum (measured in the laboratory) to evaluate the accuracy of the model.

The interlaboratory study was accomplished to measure the accuracy (reproducibility and repeatability) of the proposed method. Repeatability is a measure repeats some number within each laboratory by the same operator and equipment, which should yield the best smallest variation among readings. Reproducibility is a measure to perform the test method in many different laboratories on the same material. Because we now have different operators, different equipment, and different environmental conditions, we should expect to have greater variability in the results from different laboratories. Repeatability and reproducibility variation are best computed as a standard deviation.

\section{Results and discussion}

\section{Natural and reagent gypsum transformation}

Table 1 shows the results obtained for water loss associated with gypsum transformation from reconstituted gypsum samples upon drying at $105{ }^{\circ} \mathrm{C}$ for $24 \mathrm{~h}$. The determined values for crystal water percentage lost upon drying using natural and reagent gypsum appear to be closed, which indicate gypsum transformation to bassanite and anhydrite has very little variations in the composition. Percentage of hygroscopic water for the non-gypsiferous sandy and clay soils were 0.27 and $8.74 \%$, respectively. Percentage of hygroscopic water for the natural pure gypsum and $\mathrm{CaSO}_{4} \cdot 2 \mathrm{H}_{2} \mathrm{O}$ reagents were 4.24 and $3.64 \%$, respectively. 
Table 1 Gypsum determination using silica-gel and acetone methods compared to the proposed method in synthetic samples

\begin{tabular}{|c|c|c|c|c|c|c|c|c|c|c|}
\hline \multirow{3}{*}{$\begin{array}{l}\text { Added } \\
\text { gypsum \% }\end{array}$} & \multicolumn{5}{|c|}{ Clay soils } & \multicolumn{5}{|c|}{ Sandy soils } \\
\hline & \multicolumn{3}{|c|}{ Silica-gel method } & \multirow{2}{*}{$\begin{array}{l}\text { Acetone } \\
\text { method }\end{array}$} & \multirow{2}{*}{$\begin{array}{l}\text { Proposed } \\
\text { method }\end{array}$} & \multicolumn{3}{|c|}{ Silica-gel method } & \multirow{2}{*}{$\begin{array}{l}\text { Acetone } \\
\text { method }\end{array}$} & \multirow{2}{*}{$\begin{array}{l}\text { Proposed } \\
\text { method }\end{array}$} \\
\hline & $\begin{array}{l}\text { Hygr. } \\
\text { water \% }\end{array}$ & $\begin{array}{l}\text { Cryst. } \\
\text { water \% }\end{array}$ & $\begin{array}{l}\text { Silica- } \\
\text { gel }\end{array}$ & & & $\begin{array}{l}\text { Hygr. } \\
\text { water \% }\end{array}$ & $\begin{array}{l}\text { Cryst. } \\
\text { water } \%\end{array}$ & $\begin{array}{l}\text { Silica- } \\
\text { gel }\end{array}$ & & \\
\hline \multicolumn{11}{|c|}{$\mathrm{CaSO}_{4} \cdot 2 \mathrm{H}_{2} \mathrm{O}$} \\
\hline 0 & 8.74 & - & - & - & - & 0.27 & - & - & - & - \\
\hline 5 & 8.50 & 1.06 & 5.27 & 4.80 & 5.18 & 0.98 & 1.04 & 5.16 & 4.88 & 5.09 \\
\hline 10 & 8.47 & 2.12 & 10.53 & 9.48 & 10.37 & 0.64 & 2.07 & 10.29 & 9.41 & 10.12 \\
\hline 15 & 8.21 & 3.22 & 16.00 & 14.32 & 15.75 & 0.81 & 3.10 & 15.42 & 14.36 & 15.16 \\
\hline 20 & 7.83 & 4.19 & 20.81 & 19.20 & 20.49 & 0.98 & 4.13 & 20.56 & 19.25 & 20.20 \\
\hline 25 & 7.72 & 5.20 & 25.84 & 24.11 & 25.43 & 1.15 & 5.20 & 25.88 & 24.26 & 25.43 \\
\hline 30 & 7.38 & 6.23 & 30.96 & 29.15 & 30.46 & 1.31 & 6.31 & 31.06 & 29.14 & 30.86 \\
\hline 35 & 7.08 & 7.27 & 36.16 & 34.12 & 35.55 & 1.47 & 7.24 & 35.98 & 34.28 & 35.40 \\
\hline 40 & 6.90 & 8.29 & 41.20 & 39.13 & 40.54 & 1.64 & 8.25 & 41.03 & 38.80 & 40.34 \\
\hline 45 & 6.52 & 9.32 & 46.31 & 44.06 & 45.57 & 1.80 & 9.28 & 46.12 & 43.72 & 45.38 \\
\hline 50 & 6.17 & 10.41 & 51.73 & 48.91 & 50.90 & 1.96 & 10.32 & 51.29 & 48.72 & 50.46 \\
\hline 55 & 5.96 & 11.93 & 56.82 & 53.80 & 58.34 & 2.12 & 11.42 & 56.77 & 53.60 & 55.84 \\
\hline 60 & 5.72 & 12.33 & 61.28 & 59.32 & 60.29 & 2.28 & 12.42 & 61.74 & 58.41 & 60.73 \\
\hline 65 & 5.50 & 13.38 & 66.51 & 63.73 & 65.43 & 2.45 & 13.44 & 66.80 & 63.88 & 65.72 \\
\hline 70 & 5.22 & 14.46 & 71.86 & 68.25 & 70.71 & 2.61 & 14.46 & 71.86 & 68.21 & 70.71 \\
\hline 75 & 5.00 & 15.44 & 76.74 & 74.00 & 75.50 & 2.78 & 15.48 & 76.94 & 73.78 & 75.70 \\
\hline 80 & 4.70 & 16.45 & 81.85 & 78.92 & 80.44 & 2.94 & 16.54 & 82.25 & 78.97 & 80.88 \\
\hline 85 & 4.43 & 17.45 & 86.74 & 83.76 & 85.33 & 3.10 & 17.48 & 86.88 & 84.12 & 85.48 \\
\hline 90 & 4.17 & 18.44 & 91.65 & 88.80 & 90.17 & 3.11 & 18.57 & 92.32 & 88.92 & 90.81 \\
\hline 95 & 3.90 & 19.31 & 95.97 & 93.76 & 94.43 & 3.47 & 19.34 & 96.17 & 93.83 & 94.57 \\
\hline 100 & 3.64 & 20.12 & 101.78 & 98.15 & 98.39 & 3.64 & 20.12 & 102.00 & 98.12 & 98.39 \\
\hline \multicolumn{11}{|c|}{ Natural gypsum } \\
\hline 0 & 8.74 & - & - & - & - & 0.27 & - & - & - & - \\
\hline 5 & 8.60 & 1.00 & 5.20 & 4.87 & 5.10 & 0.49 & 1.00 & 5.19 & 4.80 & 5.09 \\
\hline 10 & 8.49 & 2.03 & 10.48 & 9.56 & 10.35 & 0.70 & 2.00 & 10.33 & 9.66 & 10.17 \\
\hline 15 & 8.14 & 3.06 & 15.82 & 14.27 & 15.61 & 0.81 & 3.01 & 15.51 & 14.47 & 15.31 \\
\hline 20 & 7.92 & 3.98 & 20.57 & 19.44 & 20.30 & 1.02 & 3.99 & 20.60 & 19.38 & 20.30 \\
\hline 25 & 7.80 & 5.02 & 25.89 & 24.62 & 25.60 & 1.23 & 5.05 & 26.04 & 24.50 & 25.69 \\
\hline 30 & 7.55 & 5.98 & 30.84 & 29.03 & 30.50 & 1.50 & 5.98 & 30.88 & 29.32 & 30.42 \\
\hline 35 & 7.25 & 6.96 & 35.91 & 33.81 & 35.50 & 1.59 & 6.99 & 36.07 & 34.29 & 35.55 \\
\hline 40 & 7.08 & 7.95 & 41.03 & 38.82 & 40.55 & 1.76 & 7.98 & 41.14 & 38.85 & 40.59 \\
\hline 45 & 6.84 & 8.94 & 46.13 & 44.02 & 45.59 & 2.00 & 8.95 & 46.19 & 43.62 & 45.52 \\
\hline 50 & 6.40 & 10.04 & 51.82 & 48.90 & 51.20 & 2.19 & 9.95 & 51.34 & 48.80 & 50.61 \\
\hline 55 & 6.36 & 11.00 & 56.79 & 53.69 & 56.10 & 2.35 & 10.98 & 56.64 & 53.73 & 55.85 \\
\hline 60 & 6.12 & 11.91 & 61.43 & 58.56 & 60.74 & 2.68 & 11.96 & 61.73 & 58.42 & 60.83 \\
\hline 65 & 5.88 & 12.98 & 66.98 & 64.35 & 66.20 & 2.87 & 12.95 & 66.80 & 63.63 & 65.87 \\
\hline 70 & 5.65 & 13.89 & 71.68 & 68.60 & 70.84 & 3.12 & 13.89 & 71.66 & 68.43 & 70.65 \\
\hline 75 & 5.31 & 14.92 & 76.93 & 73.38 & 76.09 & 3.24 & 14.90 & 76.87 & 73.36 & 75.79 \\
\hline 80 & 5.02 & 15.98 & 82.42 & 78.26 & 81.50 & 3.40 & 15.89 & 81.97 & 78.21 & 80.82 \\
\hline 85 & 4.74 & 16.75 & 86.39 & 83.60 & 85.43 & 3.63 & 16.84 & 86.86 & 83.84 & 85.66 \\
\hline 90 & 4.52 & 17.79 & 91.75 & 88.32 & 90.73 & 3.72 & 17.81 & 91.85 & 88.70 & 90.59 \\
\hline 95 & 4.47 & 18.58 & 95.83 & 90.18 & 94.76 & 4.10 & 18.51 & 95.94 & 93.75 & 94.15 \\
\hline 100 & 4.24 & 19.39 & 101.89 & 92.78 & 98.89 & 4.24 & 19.39 & 101.58 & 98.48 & 98.63 \\
\hline
\end{tabular}


Table 2 Gypsum content as determined by acetone, silica-gel methods compared with the proposed method

\begin{tabular}{|c|c|c|c|c|c|c|c|c|c|c|}
\hline \multirow[t]{2}{*}{ Soil no. } & \multicolumn{3}{|c|}{ Soil separates, \% } & \multirow{2}{*}{$\begin{array}{l}\text { Hygroscopic } \\
\text { water, \% }\end{array}$} & \multicolumn{3}{|c|}{ Silica-gel method } & \multirow[t]{2}{*}{ Gypsum, $\%$ acetone } & \multicolumn{2}{|c|}{ Gypsum, \% } \\
\hline & Sand & Silt & Clay & & $\begin{array}{l}\text { Crystal } \\
\text { water, \% }\end{array}$ & $\begin{array}{l}\text { Moisture } \\
\text { content }\end{array}$ & $\begin{array}{l}\text { Gypsum, } \\
\%\end{array}$ & & $\begin{array}{l}\text { Proposed } \\
\text { method }\end{array}$ & $\begin{array}{l}\text { Regression } \\
\text { equation }\end{array}$ \\
\hline 1 & 83.00 & 5.00 & 12.00 & 2.30 & 0.75 & 3.05 & 3.86 & 3.40 & 3.81 & 4.14 \\
\hline 2 & 39.40 & 30.00 & 30.60 & 6.45 & 2.61 & 9.06 & 13.46 & 13.23 & 13.28 & 13.61 \\
\hline 3 & 89.20 & 7.20 & 3.60 & 1.20 & 4.65 & 5.85 & 23.98 & 22.32 & 23.65 & 23.52 \\
\hline 4 & 90.00 & 6.50 & 3.50 & 0.58 & 0.20 & 0.78 & 1.03 & 0.93 & 1.02 & 1.37 \\
\hline 5 & 84.00 & 10.20 & 5.80 & 1.95 & 4.55 & 6.50 & 23.46 & 22.70 & 23.14 & 23.19 \\
\hline 6 & 81.10 & 10.30 & 8.60 & 1.72 & 3.34 & 5.06 & 17.22 & 15.13 & 16.99 & 16.90 \\
\hline 7 & 69.50 & 11.50 & 19.00 & 3.60 & 0.17 & 3.77 & 0.88 & 0.57 & 0.86 & 1.29 \\
\hline 8 & 30.50 & 38.50 & 31.00 & 6.32 & 1.04 & 7.36 & 5.36 & 4.26 & 5.29 & 5.79 \\
\hline 9 & 93.00 & 5.20 & 1.80 & 0.72 & 2.59 & 3.31 & 13.35 & 12.21 & 13.17 & 13.32 \\
\hline 10 & 20.00 & 21.80 & 58.20 & 11.59 & 0.81 & 12.40 & 4.17 & 4.12 & 4.12 & 4.78 \\
\hline 11 & 91.20 & 3.60 & 5.20 & 1.65 & 3.40 & 4.75 & 15.98 & 15.23 & 17.29 & 17.44 \\
\hline 12 & 83.00 & 8.40 & 8.60 & 2.32 & 5.33 & 7.65 & 27.48 & 25.83 & 27.11 & 26.96 \\
\hline 13 & 44.20 & 34.60 & 29.20 & 4.80 & 3.56 & 8.36 & 18.36 & 16.88 & 18.11 & 17.66 \\
\hline 14 & 24.00 & 21.00 & 55.00 & 11.92 & 4.97 & 16.89 & 25.63 & 25.43 & 25.28 & 25.65 \\
\hline 15 & 3.00 & 29.00 & 52.00 & 11.42 & 4.73 & 16.15 & 24.39 & 24.87 & 24.06 & 24.49 \\
\hline 16 & 90.40 & 5.60 & 4.00 & 0.78 & 0.40 & 1.18 & 2.06 & 2.10 & 2.03 & 2.40 \\
\hline 17 & 76.40 & 17.30 & 6.30 & 1.30 & 1.60 & 2.90 & 8.25 & 7.82 & 8.14 & 8.34 \\
\hline 18 & 78.80 & 15.20 & 6.00 & 1.28 & 1.48 & 2.76 & 7.63 & 7.21 & 7.53 & 7.77 \\
\hline 19 & 90.30 & 6.60 & 3.10 & 0.58 & 0.68 & 1.26 & 3.51 & 3.12 & 3.46 & 3.76 \\
\hline 20 & 74.00 & 20.20 & 5.80 & 1.05 & 1.23 & 2.28 & 6.34 & 5.00 & 6.26 & 6.45 \\
\hline 21 & 29.20 & 38.20 & 32.60 & 6.94 & 2.73 & 9.67 & 14.07 & 13.80 & 13.89 & 14.25 \\
\hline 22 & 83.20 & 5.60 & 11.20 & 2.36 & 1.78 & 4.14 & 9.17 & 8.56 & 9.05 & 9.30 \\
\hline 23 & 77.20 & 9.30 & 13.50 & 2.67 & 1.93 & 4.60 & 9.95 & 8.78 & 9.82 & 9.98 \\
\hline 24 & 86.00 & 11.50 & 2.50 & 0.43 & 0.31 & 0.74 & 1.60 & 1.53 & 1.58 & 1.92 \\
\hline 25 & 69.90 & 9.50 & 20.60 & 3.84 & 0.16 & 4.00 & 0.83 & 0.63 & 0.81 & 1.22 \\
\hline 26 & 74.20 & 8.60 & 17.00 & 3.88 & 2.60 & 6.48 & 13.40 & 13.82 & 13.22 & 13.53 \\
\hline 27 & 80.00 & 10.20 & 9.80 & 3.22 & 6.70 & 9.92 & 34.55 & 33.63 & 34.08 & 34.00 \\
\hline 28 & 89.80 & 6.00 & 4.20 & 1.18 & 3.16 & 4.34 & 16.29 & 16.40 & 16.07 & 16.14 \\
\hline 29 & 85.20 & 13.20 & 1.60 & 1.66 & 6.71 & 8.37 & 34.61 & 32.52 & 34.13 & 34.03 \\
\hline 30 & 12.00 & 25.00 & 53.00 & 12.61 & 1.04 & 13.65 & 5.35 & 5.10 & 5.29 & 6.79 \\
\hline 31 & 13.00 & 27.00 & 60.00 & 12.14 & 0.98 & 13.12 & 5.05 & 4.62 & 4.98 & 5.71 \\
\hline 32 & 30.00 & 22.00 & 48.00 & 9.57 & 1.00 & 10.57 & 5.15 & 4.20 & 5.09 & 5.65 \\
\hline 33 & 12.00 & 40.00 & 48.00 & 9.58 & 1.05 & 10.63 & 5.42 & 4.30 & 5.34 & 5.90 \\
\hline 34 & 83.00 & 9.60 & 7.40 & 1.39 & 0.20 & 1.59 & 1.03 & 0.80 & 1.02 & 1.41 \\
\hline 35 & 62.50 & 21.00 & 16.50 & 3.30 & 0.75 & 4.05 & 3.86 & 3.72 & 3.81 & 4.21 \\
\hline 36 & 70.00 & 10.60 & 19.40 & 3.82 & 0.51 & 4.33 & 2.63 & 2.43 & 2.59 & 3.03 \\
\hline 37 & 62.00 & 15.50 & 22.50 & 4.72 & 2.04 & 6.76 & 10.52 & 9.32 & 10.38 & 10.70 \\
\hline 38 & 84.90 & 4.50 & 10.60 & 1.97 & 2.43 & 4.40 & 12.53 & 10.12 & 12.36 & 12.37 \\
\hline 39 & 80.50 & 5.30 & 14.20 & 2.86 & 1.55 & 4.41 & 7.99 & 7.32 & 7.88 & 8.14 \\
\hline 40 & 84.50 & 8.30 & 7.20 & 1.36 & 0.57 & 1.93 & 2.93 & 2.65 & 2.90 & 3.23 \\
\hline 41 & 25.50 & 32.50 & 42.00 & 8.44 & 1.16 & 9.60 & 5.98 & 6.24 & 5.90 & 6.43 \\
\hline 42 & 86.50 & 7.30 & 6.20 & 1.84 & 6.36 & 8.20 & 32.80 & 31.73 & 32.35 & 32.01 \\
\hline 43 & 78.00 & 8.50 & 13.50 & 2.86 & 2.45 & 5.31 & 12.63 & 11.22 & 12.46 & 12.63 \\
\hline 44 & 88.00 & 6.40 & 5.60 & 1.63 & 3.58 & 5.21 & 18.46 & 17.40 & 18.21 & 18.29 \\
\hline 45 & 70.00 & 12.20 & 17.80 & 3.91 & 3.34 & 7.25 & 17.22 & 15.48 & 16.99 & 17.12 \\
\hline 46 & 86.50 & 6.30 & 7.20 & 1.80 & 2.80 & 4.60 & 14.44 & 13.82 & 14.24 & 14.39 \\
\hline
\end{tabular}


Table 2 continued

\begin{tabular}{|c|c|c|c|c|c|c|c|c|c|c|c|c|}
\hline \multirow[t]{2}{*}{ Soil no. } & \multicolumn{4}{|c|}{ Soil separates, $\%$} & \multirow{2}{*}{$\begin{array}{l}\text { Hygroscopic } \\
\text { water, \% }\end{array}$} & \multicolumn{3}{|c|}{ Silica-gel method } & \multirow{2}{*}{\multicolumn{2}{|c|}{ Gypsum, \% acetone }} & \multicolumn{2}{|c|}{ Gypsum, \% } \\
\hline & \multicolumn{2}{|c|}{ Sand } & Silt & Clay & & $\begin{array}{l}\text { Crystal } \\
\text { water, \% }\end{array}$ & $\begin{array}{l}\text { Moisture } \\
\text { content }\end{array}$ & $\begin{array}{l}\text { Gypsum, } \\
\%\end{array}$ & & & $\begin{array}{l}\text { Proposed } \\
\text { method }\end{array}$ & $\begin{array}{l}\text { Regression } \\
\text { equation }\end{array}$ \\
\hline 47 & \multicolumn{2}{|c|}{48.40} & 25.20 & 26.40 & 5.17 & 0.76 & 5.93 & 3.92 & \multicolumn{2}{|l|}{3.28} & 3.87 & 4.28 \\
\hline 48 & \multicolumn{2}{|c|}{89.30} & 6.80 & 3.90 & 0.73 & 1.91 & 2.64 & 9.85 & \multicolumn{2}{|l|}{8.46} & 9.72 & 9.81 \\
\hline 49 & 88. & & 6.60 & 5.00 & 1.02 & 0.72 & 1.74 & 3.71 & 3.62 & & 3.66 & 4.00 \\
\hline 50 & 87. & & 9.50 & 3.00 & 0.52 & 0.38 & 0.90 & 1.95 & 1.73 & & 1.93 & 2.27 \\
\hline 51 & 91. & & 6.20 & 2.10 & 0.36 & 0.22 & 0.58 & 1.13 & 1.03 & & 1.12 & 1.48 \\
\hline 52 & 68. & & 2.50 & 8.60 & 2.12 & 3.98 & 6.10 & 20.52 & 19.80 & & 20.24 & 20.23 \\
\hline 53 & 75. & & 0.20 & 14.50 & 4.24 & 7.77 & 12.01 & 40.17 & 38.48 & & 39.52 & 39.33 \\
\hline 54 & 24. & & 30.40 & 45.60 & 9.32 & 1.32 & 10.64 & 6.80 & 6.43 & & 6.71 & 7.31 \\
\hline 55 & 20. & & 26.80 & 52.30 & 11.18 & 3.30 & 14.48 & 17.01 & 16.70 & & 16.79 & 17.33 \\
\hline 56 & 23. & & 10.70 & 36.00 & 7.45 & 2.02 & 9.47 & 10.41 & 10.12 & & 10.27 & 10.71 \\
\hline 57 & 30. & & 34.30 & 35.20 & 7.03 & 1.14 & 8.17 & 5.87 & 5.22 & & 5.80 & 6.26 \\
\hline 58 & 71. & & 5.30 & 13.60 & 1.75 & 3.36 & 5.09 & 17.28 & 15.20 & & 16.99 & 16.60 \\
\hline 59 & 54. & & 25.20 & 20.80 & 1.01 & 1.26 & 2.22 & 6.39 & 5.00 & & 6.26 & 5.35 \\
\hline 60 & 80. & & 9.80 & 9.90 & 0.72 & 1.90 & 2.60 & 9.80 & 8.58 & & 9.72 & 9.26 \\
\hline Soil & Soil se & parates, & $\%$ & Hygrc & copic & Silica-gel met & od & & Gypsum, \% & Gypsum & $\mathrm{n}, \%$ & \\
\hline . & Sand & Silt & Clay & & & $\begin{array}{l}\text { Crystal } \\
\text { water, \% }\end{array}$ & $\begin{array}{l}\text { Moisture } \\
\text { content }\end{array}$ & $\begin{array}{l}\text { Gypsum, } \\
\%\end{array}$ & & $\begin{array}{l}\text { Proposec } \\
\text { method }\end{array}$ & & $\begin{array}{l}\text { Regression } \\
\text { equation }\end{array}$ \\
\hline 61 & 89.40 & 6.90 & 3.70 & 0.53 & & 0.23 & 0.76 & 1.08 & 0.97 & 1.17 & & 1.47 \\
\hline 62 & 80.50 & 10.70 & 8.80 & 1.67 & & 3.37 & 5.04 & 17.27 & 15.17 & 17.14 & & 17.01 \\
\hline 63 & 29.90 & 38.90 & 31.20 & 6.27 & & 1.07 & 7.34 & 5.41 & 4.30 & 5.44 & & 5.90 \\
\hline 64 & 90.60 & 4.00 & 5.40 & 1.60 & & 3.43 & 5.03 & 16.03 & 15.27 & 17.45 & & 17.55 \\
\hline 65 & 43.60 & 35.00 & 29.40 & 4.75 & & 3.59 & 8.34 & 18.41 & 16.92 & 18.26 & & 17.77 \\
\hline 66 & 38.80 & 30.40 & 30.80 & 6.40 & & 2.64 & 9.04 & 13.51 & 13.27 & 13.43 & & 13.72 \\
\hline 67 & 83.40 & 10.60 & 6.00 & 1.90 & & 4.58 & 6.48 & 23.51 & 22.74 & 23.30 & & 23.29 \\
\hline 68 & 89.80 & 6.00 & 4.20 & 0.73 & & 0.43 & 1.16 & 2.11 & 2.14 & 2.19 & & 2.51 \\
\hline 69 & 29.40 & 22.40 & 48.20 & 9.52 & & 1.03 & 10.55 & 5.20 & 4.24 & 5.24 & & 5.76 \\
\hline 70 & 23.40 & 21.40 & 55.20 & 11.87 & & 5.00 & 16.87 & 25.68 & 25.47 & 25.43 & & 25.76 \\
\hline 71 & 2.40 & 29.40 & 52.20 & 11.37 & & 4.76 & 16.13 & 24.44 & 24.91 & 24.21 & & 24.60 \\
\hline 72 & 92.40 & 5.60 & 2.00 & 0.67 & & 2.62 & 3.29 & 13.40 & 12.25 & 13.33 & & 13.43 \\
\hline 73 & 19.40 & 22.20 & 58.40 & 11.54 & & 0.84 & 12.38 & 4.22 & 4.16 & 4.27 & & 4.88 \\
\hline 74 & 82.40 & 8.80 & 8.80 & 2.27 & & 5.36 & 7.63 & 27.53 & 25.87 & 27.26 & & 27.07 \\
\hline 75 & 90.00 & 6.70 & 3.30 & 1.15 & & 4.68 & 5.83 & 24.03 & 22.36 & 23.80 & & 23.67 \\
\hline 76 & 83.80 & 4.50 & 11.70 & 2.25 & & 0.78 & 3.03 & 3.83 & 3.42 & 3.97 & & 4.29 \\
\hline 77 & 13.80 & 26.50 & 59.70 & 12.09 & & 1.01 & 13.10 & 5.02 & 4.64 & 5.14 & & 5.86 \\
\hline 78 & 82.70 & 5.80 & 11.50 & 2.31 & & 1.81 & 4.12 & 9.14 & 8.58 & 9.21 & & 9.40 \\
\hline 79 & 77.20 & 16.80 & 6.00 & 1.25 & & 1.63 & 2.88 & 8.22 & 7.84 & 8.29 & & 8.49 \\
\hline 80 & 78.00 & 8.80 & 13.20 & 2.69 & & 1.91 & 4.60 & 9.92 & 8.80 & 9.72 & & 9.92 \\
\hline 81 & 70.70 & 9.00 & 20.30 & 3.86 & & 0.14 & 4.00 & 0.80 & 0.65 & 0.71 & & 1.15 \\
\hline 82 & 79.60 & 14.70 & 5.70 & 1.30 & & 1.46 & 2.76 & 7.60 & 7.23 & 7.43 & & 7.70 \\
\hline 83 & 91.10 & 6.10 & 2.80 & 0.60 & & 0.66 & 1.26 & 3.48 & 3.14 & 3.36 & & 3.69 \\
\hline 84 & 86.00 & 12.70 & 1.30 & 1.68 & & 6.69 & 8.37 & 34.58 & 32.54 & 34.03 & & 33.96 \\
\hline 85 & 12.80 & 24.50 & 52.70 & 12.63 & & 1.02 & 13.65 & 5.32 & 5.12 & 5.19 & & 6.73 \\
\hline 86 & 86.80 & 11.00 & 2.20 & 0.45 & & 0.29 & 0.74 & 1.57 & 1.55 & 1.48 & & 1.86 \\
\hline 87 & 92.50 & 5.70 & 1.80 & 0.38 & & 0.20 & 0.58 & 1.10 & 1.05 & 1.02 & & 1.42 \\
\hline 88 & 12.80 & 39.50 & 47.70 & 9.60 & & 1.03 & 10.63 & 5.39 & 4.32 & 5.24 & & 5.83 \\
\hline 89 & 61.50 & 15.70 & 22.80 & 4.74 & & 2.02 & 6.76 & 10.49 & 9.34 & 10.27 & & 10.59 \\
\hline
\end{tabular}


Table 2 continued

\begin{tabular}{|c|c|c|c|c|c|c|c|c|c|c|}
\hline \multirow{2}{*}{$\begin{array}{l}\text { Soil } \\
\text { no. }\end{array}$} & \multicolumn{3}{|c|}{ Soil separates, \% } & \multirow{2}{*}{$\begin{array}{l}\text { Hygroscopic } \\
\text { water, \% }\end{array}$} & \multicolumn{3}{|c|}{ Silica-gel method } & \multirow{2}{*}{$\begin{array}{l}\text { Gypsum, \% } \\
\text { acetone }\end{array}$} & \multicolumn{2}{|c|}{ Gypsum, \% } \\
\hline & Sand & Silt & Clay & & $\begin{array}{l}\text { Crystal } \\
\text { water, \% }\end{array}$ & $\begin{array}{l}\text { Moisture } \\
\text { content }\end{array}$ & $\begin{array}{l}\text { Gypsum, } \\
\%\end{array}$ & & $\begin{array}{l}\text { Proposed } \\
\text { method }\end{array}$ & $\begin{array}{l}\text { Regression } \\
\text { equation }\end{array}$ \\
\hline 90 & 83.80 & 9.10 & 7.10 & 1.41 & 0.18 & 1.59 & 1.00 & 0.82 & 0.92 & 1.34 \\
\hline 91 & 28.70 & 38.40 & 32.90 & 6.96 & 2.71 & 9.67 & 14.04 & 13.82 & 13.78 & 14.14 \\
\hline 92 & 70.30 & 11.00 & 18.70 & 3.62 & 0.15 & 3.77 & 0.85 & 0.59 & 0.76 & 1.22 \\
\hline 93 & 73.70 & 8.80 & 17.30 & 3.90 & 2.58 & 6.48 & 13.37 & 13.84 & 13.12 & 13.42 \\
\hline 94 & 24.80 & 29.90 & 45.30 & 9.34 & 1.30 & 10.64 & 6.84 & 6.46 & 6.61 & 7.24 \\
\hline 95 & 89.30 & 6.20 & 4.50 & 1.20 & 3.14 & 4.34 & 16.33 & 16.43 & 15.97 & 16.02 \\
\hline 96 & 62.00 & 21.20 & 16.80 & 3.32 & 0.73 & 4.05 & 3.90 & 3.75 & 3.71 & 4.10 \\
\hline 97 & 69.50 & 10.80 & 19.70 & 3.84 & 0.49 & 4.33 & 2.67 & 2.46 & 2.49 & 2.91 \\
\hline 98 & 87.00 & 9.70 & 3.30 & 0.54 & 0.36 & 0.90 & 1.99 & 1.76 & 1.83 & 2.15 \\
\hline 99 & 68.40 & 22.70 & 8.90 & 2.13 & 3.97 & 6.10 & 20.56 & 19.83 & 20.19 & 20.16 \\
\hline 100 & 25.00 & 32.70 & 42.30 & 8.45 & 1.15 & 9.60 & 6.02 & 6.27 & 5.85 & 6.36 \\
\hline 101 & 74.80 & 10.40 & 14.80 & 4.25 & 7.76 & 12.01 & 40.21 & 38.51 & 39.47 & 39.26 \\
\hline 102 & 20.40 & 27.00 & 52.60 & 11.19 & 3.29 & 14.48 & 17.05 & 16.73 & 16.73 & 17.26 \\
\hline 103 & 22.80 & 40.90 & 36.30 & 7.46 & 2.01 & 9.47 & 10.45 & 10.15 & 10.22 & 10.64 \\
\hline 104 & 79.50 & 10.40 & 10.10 & 3.23 & 6.69 & 9.92 & 34.59 & 33.66 & 34.03 & 33.93 \\
\hline 105 & 74.80 & 19.70 & 5.50 & 1.06 & 1.22 & 2.28 & 6.38 & 5.03 & 6.21 & 6.43 \\
\hline 106 & 71.50 & 15.20 & 13.30 & 1.76 & 3.35 & 5.11 & 17.32 & 15.23 & 16.99 & 16.58 \\
\hline 107 & 87.50 & 6.60 & 5.90 & 1.64 & 3.57 & 5.21 & 18.50 & 17.43 & 18.16 & 18.22 \\
\hline 108 & 69.50 & 12.40 & 18.10 & 3.92 & 3.33 & 7.25 & 17.26 & 15.51 & 16.94 & 17.05 \\
\hline 109 & 80.00 & 5.50 & 14.50 & 2.87 & 1.54 & 4.41 & 8.03 & 7.35 & 7.83 & 8.07 \\
\hline 110 & 89.70 & 6.70 & 3.60 & 0.74 & 1.90 & 2.64 & 9.89 & 8.49 & 9.66 & 9.79 \\
\hline 111 & 80.70 & 9.70 & 9.60 & 0.73 & 1.89 & 2.62 & 9.76 & 8.55 & 9.72 & 9.24 \\
\hline 112 & 84.90 & 8.20 & 6.90 & 1.37 & 0.56 & 1.93 & 2.89 & 2.62 & 2.85 & 3.21 \\
\hline 113 & 86.90 & 7.20 & 5.90 & 1.85 & 6.35 & 8.20 & 32.76 & 31.70 & 32.30 & 31.99 \\
\hline 114 & 78.40 & 8.40 & 13.20 & 2.87 & 2.44 & 5.31 & 12.59 & 11.19 & 12.41 & 12.61 \\
\hline 115 & 54.40 & 25.10 & 20.50 & 0.98 & 1.24 & 2.22 & 6.35 & 4.97 & 6.26 & 5.26 \\
\hline 116 & 30.90 & 34.20 & 34.90 & 7.00 & 1.12 & 8.12 & 5.83 & 5.24 & 5.70 & 6.17 \\
\hline 117 & 86.90 & 6.20 & 6.90 & 1.77 & 2.78 & 4.55 & 14.40 & 13.79 & 14.14 & 14.31 \\
\hline 118 & 87.90 & 6.80 & 5.30 & 0.99 & 0.70 & 1.69 & 3.67 & 3.59 & 3.56 & 3.86 \\
\hline 119 & 85.30 & 4.40 & 10.30 & 1.94 & 2.41 & 4.35 & 12.49 & 10.09 & 12.26 & 12.28 \\
\hline 120 & 48.80 & 25.10 & 26.10 & 5.14 & 0.74 & 5.88 & 3.88 & 3.25 & 3.76 & 4.20 \\
\hline
\end{tabular}

It appears from the results that gypsum transformation to bassanite and anhydrite show very little variations in the composition of gypsum. The difference is mainly in its chemical composition as a result of its dehydration upon heating. Crystal water of natural gypsum and reagent used is constitutes 19.39 and $20.12 \%$ of the total weight, respectively. This means that gypsum decomposes upon heating to bassanite and finally to anhydrite, loss average $19.76 \%$ of its total weight.

\section{Gypsum content determination in soil samples}

To test the validity of Eq. 1, results from the silica-gel, acetone, and the proposed method were compared
(Table 2) for 120 samples. Regression equation (Eq. 2) indicated that the calculated values are highly correlated and overestimation of gypsum content compared with acetone method, whereas underestimation compared with silica-gel method. With this closed agreement, we suggest that this equation could be used with good accuracy for predicting gypsum content in soils.

$\mathrm{Y}=-0.3008+4.919 \mathrm{X}_{1}+0.5566 \mathrm{X}_{2}-0.0724 \mathrm{X}_{3}$.

where, $\mathrm{Y}=$ Gypsum content, $\mathrm{X}_{1}=$ Crystal water, $\mathrm{X}_{2}$ $=$ Hygroscopic water, $\mathrm{X}_{3}=$ Clay content.

The results indicate that percent total water loss associated with drying gypsiferous soil samples is not only 

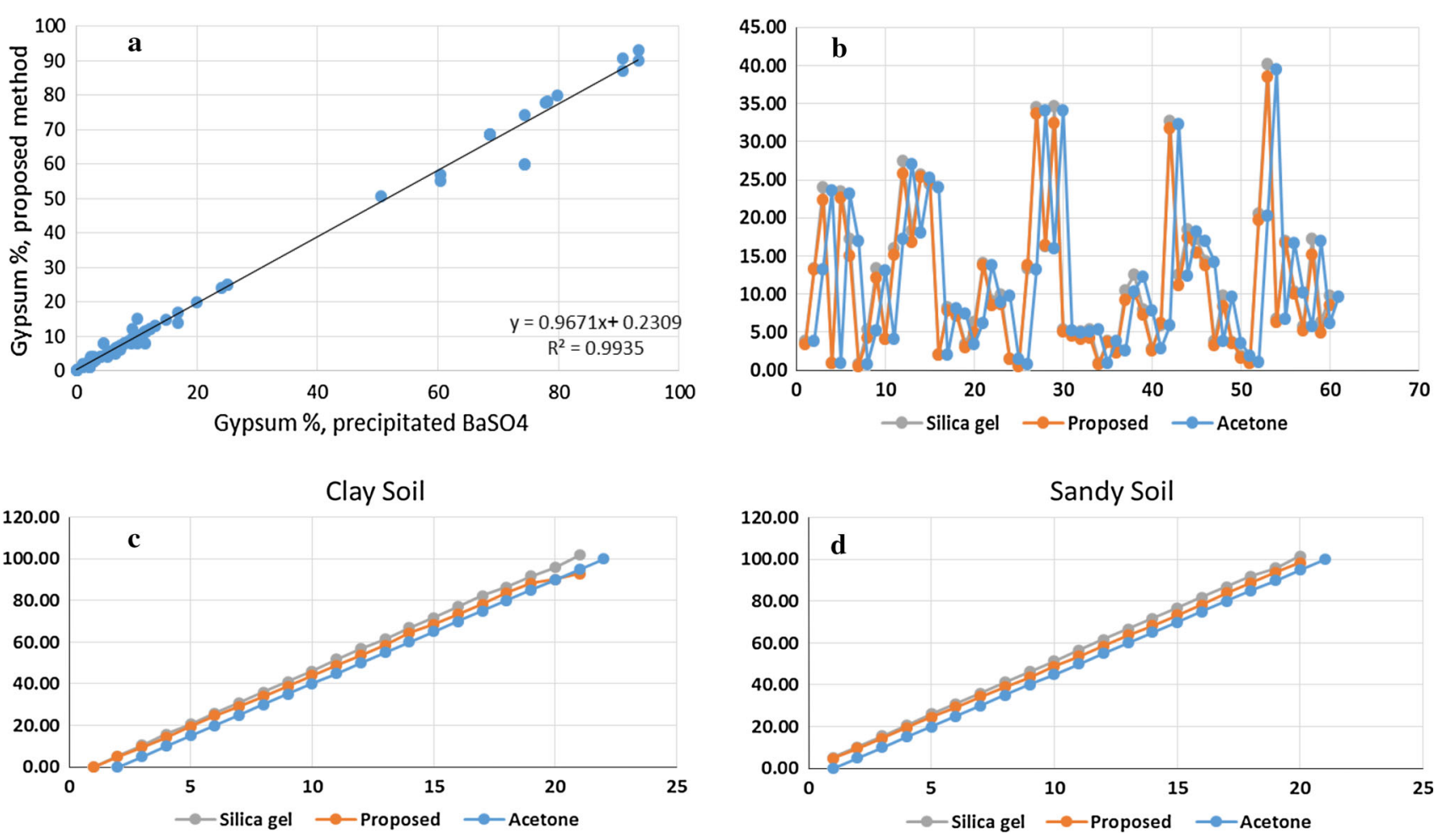

Fig. 2 Gypsum content determined from precipitated $\mathrm{BaSO}_{4}$ as related to gypsum content from the proposed method and accuracy of proposed method compared with other methods

Table 3 Recovery of known pure gypsum amount added to soil by silica-gel, acetone, and the proposed method

\begin{tabular}{|c|c|c|c|c|c|c|}
\hline \multirow[t]{2}{*}{ Gypsum, \% } & \multicolumn{2}{|c|}{ Proposed } & \multicolumn{2}{|c|}{ Silica-gel } & \multicolumn{2}{|c|}{ Acetone } \\
\hline & Mean & SD & Mean & SD & Mean & SD \\
\hline 5 & 5.14 & 0.06 & 6.04 & 0.31 & 4.04 & 0.43 \\
\hline 10 & 10.06 & 0.06 & 10.96 & 0.31 & 8.96 & 0.43 \\
\hline 20 & 20.25 & 0.05 & 21.15 & 0.30 & 19.15 & 0.42 \\
\hline 30 & 30.78 & 0.08 & 31.68 & 0.33 & 29.68 & 0.45 \\
\hline 40 & 40.42 & 0.08 & 41.32 & 0.33 & 39.32 & 0.45 \\
\hline 50 & 50.63 & 0.17 & 51.53 & 0.42 & 49.53 & 0.54 \\
\hline 60 & 60.72 & 0.02 & 61.62 & 0.27 & 59.62 & 0.39 \\
\hline 70 & 70.65 & 0.05 & 71.55 & 0.30 & 69.55 & 0.42 \\
\hline 80 & 80.84 & 0.04 & 81.74 & 0.29 & 79.74 & 0.41 \\
\hline 90 & 90.80 & 0.01 & 91.70 & 0.25 & 89.70 & 0.37 \\
\hline 100 & 98.89 & 0.49 & 99.79 & 0.74 & 97.79 & 0.86 \\
\hline
\end{tabular}

$S D$ standard deviation

dependent on soil texture and its clay content, but also on percent gypsum. However, the rate of gypsum dehydration is catalyzed by soil matrix nature (Elprince et al. 1982). Clay minerals contain a considerable amount of crystal water. During the determination of the crystal water in gypsum by the classical thermal analysis method, not only the removal of the crystal water occurs, but also, the dehydration of the clay minerals occurs. To prevent errors arising in the determination of the crystal water in gypsum, first, it is necessary to dry the gypsum samples to constant mass at $50{ }^{\circ} \mathrm{C}$ in an electric dryer. Crystal water of pure gypsum is about $19.76 \%$ of its total weight. This means that when gypsum decomposes upon heating to bassanite and finally to anhydrite, loses about $19.76 \%$ of its total weight. Accordingly, the properties of gypsiferous soils on an oven-dry weight basis should be corrected to include gypsum crystal water. Further, it is necessary to avoid the possible calculation error when the data are expressed on an oven-dry weight basis that the loss of gypsum crystal water must be subtracted from the total soil moisture content if determined by drying at $150{ }^{\circ} \mathrm{C}$.

\section{An accuracy assessment of the proposed method}

Gypsum content (Fig. 2a) calculated in the proposed method was found to be highly correlated $\left(r^{2}=0.99\right)$ with those determined by the gravimetry of precipitated $\mathrm{BaSO}_{4}$. For testing the accuracies of the Eq. 2, gypsum content is calculated for the soils (Fig. 2b) and for the artificial gypsum (Fig. 2c, d). Regression equation indicates that the calculated values overestimate the gypsum compared with added gypsum and the proposed OMRAN GypSim method.

Table 3 shows the average percentage of added gypsum retrieved by the three methods indicates that the reproducibility of the proposed method is the highest, which are 
ranged between 0.01 and 0.49 (average 0.10 ) followed by silica-gel, which is ranged between 0.25 and 0.74 (average $0.35)$ then the acetone method, which is ranged between 0.37 and 0.86 (average 0.47). This simple, yet effective method determined gypsum content to within $1 \%$, whereas other methods overestimated gypsum content by as much as $10 \%$.

\section{Modelling the proposed method}

Statistical analysis based on Table 2 shows positive and the highly correlation between hygroscopic water and clay content. The prediction equation can be written as follows:

$\mathrm{Y}=(\mathrm{Y} 1+\mathrm{Y} 2)+\mathrm{Y} 3$.

where $\mathrm{Y}=$ total moisture content, $\mathrm{Y} 1$ and $\mathrm{Y} 2=$ hygroscopic water of soil and gypsum, respectively, Y3 = crystal water of gypsum.

$\mathrm{Y} 1=0.1998 \mathrm{X} 1-0.2821\left(\mathrm{r}^{2}=0.98\right)$.

where $\mathrm{Y} 1$ = hygroscopic water of soil, $\mathrm{X} 1$ = clay content.

$\mathrm{Y} 2=0.0424 \mathrm{X} 2\left(\mathrm{r}^{2}=0.93\right)$.

where $\mathrm{Y} 2$ = hygroscopic water of gypsum, $\mathrm{X} 2$ = gypsum content.

Also, there is a positive and high significant relation between gypsum content and crystal water. The stepwise equation that governs this relation can be summarized as:

$\mathrm{Y} 3=0.1938 \mathrm{X} 2-0.0065\left(\mathrm{r}^{2}=0.99\right)$.

From the above equations, the gypsum content can be derived as follows:

$\mathrm{Y}=0.1998 \mathrm{X} 1+0.2362 \mathrm{X} 2-0.2761$.

where $\mathrm{Y}=$ total moisture content, $\mathrm{X} 1=$ clay content, $\mathrm{X} 2$ = gypsum content

$\mathrm{X} 2=\mathrm{Y}-0.1998 \mathrm{X} 1+0.2761 / 0.2362$.

So, Eq. 8 may be corrected by approximately $3.6 \%$ (deviate values from the standard) as follows:

$\mathrm{X} 2=\mathrm{Y}-0.1998 \mathrm{X} 1+0.2761 / 0.2362 \times 0.964$.

A gypsum simulation (OMRAN GypSim) model is developed and implemented for gypsum determination. The OMRAN GypSim is a tool written in the language Java and is used to calculate gypsum content in arid soils. Java is one of the most popular programming languages in use. An easy-to-use graphical user interface (GUI) has been designed for the running of the model. The GUI allows users to interact with the computer hardware in a userfriendly way. In the OMRAN GypSim menu (Fig. 3) the user selects the determination methods and the parameters,

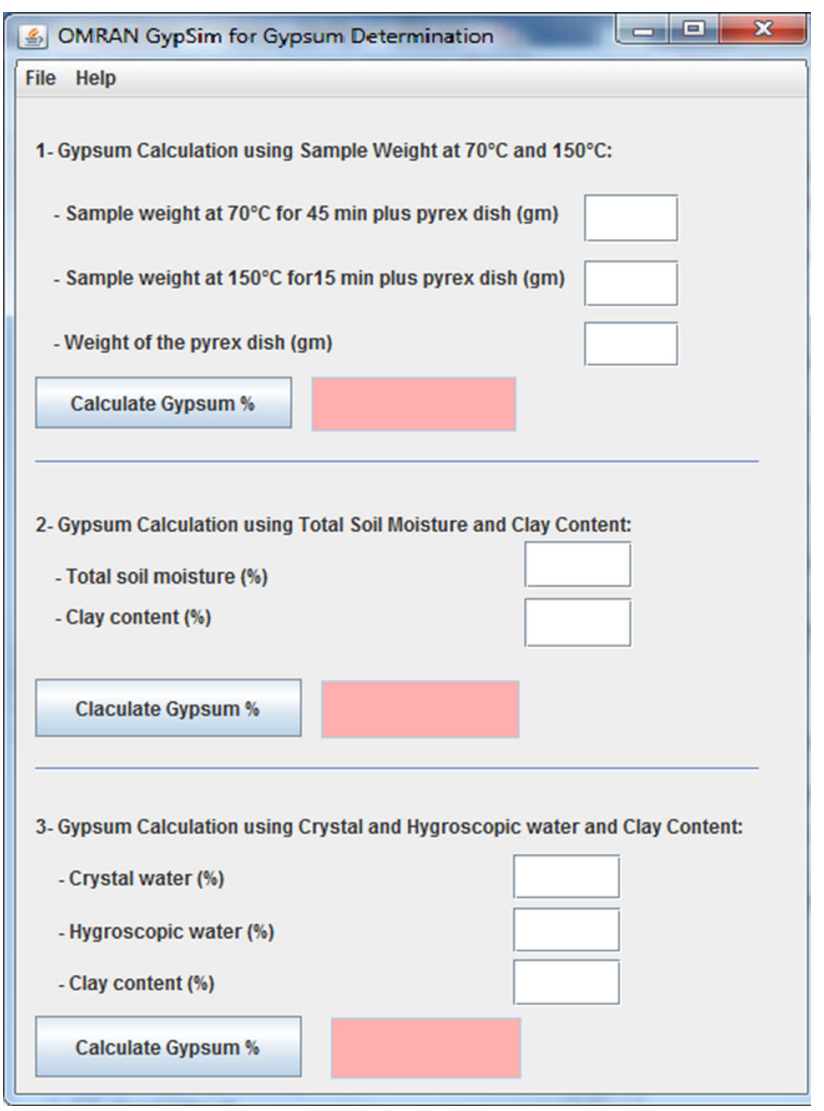

Fig. 3 OMRAN GypSim application menu to calculate gypsum content in arid soils

that is, hygroscopic and crystal water, soil moisture, and clay content. A screenshot of the application model to calculate and display the gypsum content is shown in Fig. 3.

\section{Benefits and advantages of the proposed OMRAN GypSim method}

There will be much debate among soil scientists regarding the best or favored methods for gypsum content determination. Researchers should keep in mind the following factors when considering the best methods:

1. Cost and simplicity. The labor cost vs. capital equipment cost is one of the main considerations when choosing methods. The proposed method has very low cost.

2. Available instrumentation. The most powerful and valuable diagnosis can be done with quite simple equipment. OMRAN GypSim method has very low infrastructure expenses.

3. Accuracy and precision requirements are generally lower in other methods than in the proposed method. Accuracy costs money and the proposed method using 
weighing and heating only, which is faster while still providing sufficient accuracy and precision for diagnostic and monitoring purposes. OMRAN GypSim method determined gypsum content to within $1 \%$, whereas other methods overestimated gypsum content by as much as $10 \%$.

4. Adaptable to various soil types. Many methods were developed for particular soil types and the calibration data may not be so relevant to other soil types. In particular, the proposed method developed and examined for different soil types (e.g., sand, clay, soil, etc.) and a wide variety of conditions (e.g., salinity, calcareous soil, alkalinity).

5. Robust and ideal for applications that require speed results and decision. The gypsum content of 120 soil samples can be determined in 60 min work.

6. An easy-to-use graphical user interface has been designed for the automation of the gypsum calculation.

\section{Conclusions}

Conventional methods of gypsum determination are timeconsuming and expensive and sometimes incorporate the use of environmentally damaging chemicals. There are many proposed methods of gypsum determination and each one was reported with its own limitations. Recovery gypsum content by using acetone methods of known amount of pure gypsum added to soil shows acetone is underestimated. This could be attributed to incomplete extraction of gypsum in acetone method, then more time consuming, and degree of accuracy. These methods seem to have many limitations, which can be attributed to the degree of accuracy and slow in processing. However, the silica-gel method gives values overestimate the gypsum content, which could be attributed to the partial dehydration and dehydroxylation of clay minerals causing overestimation of gypsum content. The speed and simplicity of analysis and the accuracy of the results are the main factors, which are considered in the proposed method. OMRAN GypSim was proposed for gypsum determination in soils. The proposed method for gypsum determination determines the water loss of a soil sample by heating at $70{ }^{\circ} \mathrm{C}$ and $135-150{ }^{\circ} \mathrm{C}$, which allows an accurate estimate of the gypsum content of a soil using a recovery factor of $19.66 \%$. In a laboratory, the gypsum content of 120 soil samples can be determined in 60 min work. Regression equation indicated that gypsum content determined by proposing method was highly correlated in a nearly 1:1 relationship. The reproducibility of the proposed method is the highest $(0.10)$ followed by silica-gel (0.35) then the acetone method (0.47). In conclusion, the proposed methods are simple, accurate, rapid, and less expensive. The results of pure gypsum content, as well as recovery of gypsum from the artificial mixture, suggest that the proposed method is recommended to be used in the arid- soil regions. This method is simple, rapid and less expensive. Also, an easy-to-use graphical user interface has been designed for the running of the model. A different approach for future rapidly characterizing soil gypsum at a large scale is offered by the remote sensing technique.

Acknowledgements The author wishes to thank Prof. Dr. M. Reda and Miss. S. M. Reda for their invaluable assistance in the proofreading of the manuscript.

\section{References}

Al-Awajy MH, Heakel MS, Reda M, Modaihsh AS, Choudhary MI (1994) Reliability of gypsum determination in soils by three existing simple methods. Arab Gulf J Sci Res 12(2):273-284

Artieda O, Herrero J, Drohan PJ (2006) Refinement of the differential water loss method for gypsum determination in soils. Soil Sci Soc Am J 70:1932-1935. doi:10.2136/sssaj2006.0043N

Burns DT, Danzer K, Townshend A (2002) Use of the terms "recovery" and "apparent recovery" in analytical procedures. Pure Appl Chem 74:2201-2205

Congalton R, Green K (1999) Assessing the accuracy of remotely sensed data: principles and practices. Lewis Publisher, Boca Raton

Elprince AM, Turjoman AM (1983) Infrared dehydration method for determining gypsum content of soils. Soil Sci Soc Am J 47:1089-1091

Elprince AM, Al-Shammary MA, Magboul AM (1982) Kinetics of thermal dehydration of gypsiferous soils. Soil Sci Aoc Am J 46:530-535

FAO (1990) Management of gypsiferous soils. Soils bulletin 62. Food and Agriculture Organization of the United Nations, Rome

Farag FM (1999) Thermal transformation of gypsum and its determination insoils. J Agric Sci Mansoura Univ 24(9):5201-5215

Herrero J, Artieda O, Weindorf DC (2016) Soil gypsum determination. Methods Soil Anal 1. doi:10.2136/methods-soil.2016.0037

Khan SU, Webster GR (1968) Determination of gypsum in solonetzic soils by an X-ray technique. Analyst 93:400-402. doi:10.1039/ AN9689300400

Lebron I, Herrero J, Robinson DA (2009) Determination of gypsum content in dryland soils exploiting the gypsum-bassanite phase change. Soil Sci Soc Am J 73:403-411

León J, Seeger M, Badía D, Peters P, Echeverría MT (2014) Thermal shock and splash effects on burned gypseous soils from the Ebro Basin (NE Spain). Solid Earth 5:131-140

Mahabadi NY, Givi J (2008) Comparision of different methods of gypsum determination in selected soils from Isfahan. J Water Soil Sci 11:565-576

Nelson RE, Klameth LC, Nettleton WD (1978) Determining soil gypsum content and expressing properties of gypsiferous soils. Soil Sci Soc Am J 42:659-661

Omran ESE (2008) Is soil science dead and buried? Future image in the world of 10 billion people. CATRINA 3(2):59-68

Omran ESE (2012) A neural network model for mapping and predicting unconventional soils at a regional level. Appl Remote Sens J 2(2):35-44 
Porta J (1998) Methodologies for the analysis and characterization of gypsum in soils: a review. Geoderma 87:31. doi:10.1016/S00167061(98)00067-6

Reda M (1995) Sinkholes formation in some gypsic soils of Egypt. J Agric Sci Mansoura Univ 20(2):915-922

Skarie RL, Arndt JL, Richardson JL (1987) Sulfate and gypsum determination in saline soils. Soil Sci Soc Am J 51:901-905

Soil Survey Staff (2010) Keys to soil taxonomy, 11th edn. SSDA NRCS US Gov Print Office, Washington, DC

US Salinity Laboratory Staff (1954) Diagnosis and improvement of saline and alkali soils. USDA Handbook 60, US Government Printing Office, Washington, D C
Visconti F, De Paz JM, Rubio JL (2010) Calcite and gypsum solubility products in water-saturated salt affected soil samples at $25{ }^{\circ} \mathrm{C}$ and at least up to $14 \mathrm{dS} \mathrm{m}-1$. Eur J Soil Sci 61:255. doi:10.1111/j.1365-2389.2009.01214.x

Weindorf DC, Bakr N, Zhu Y (2014) Advances in portable X-ray fluorescence (PXRF) for environmental, pedological, and agronomic applications. Adv Agron 128:1-45. doi:10.1016/B978-012-802139-2.00001-9 